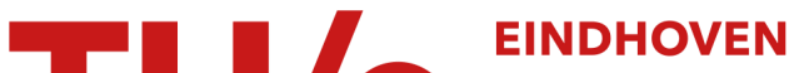 \\ UNIVERSITY OF \\ TECHNOLOGY
}

\section{ß-plane turbulence in a basin with no-slip boundaries}

Citation for published version (APA):

Kramer, W., van Buren, M. G., Clercx, H. J. H., \& Heijst, van, G. J. F. (2006). ß-plane turbulence in a basin with no-slip boundaries. Physics of Fluids, 18(2), 026603-1/11. https://doi.org/10.1063/1.2173285

DOI:

10.1063/1.2173285

Document status and date:

Published: 01/01/2006

\section{Document Version:}

Publisher's PDF, also known as Version of Record (includes final page, issue and volume numbers)

\section{Please check the document version of this publication:}

- A submitted manuscript is the version of the article upon submission and before peer-review. There can be important differences between the submitted version and the official published version of record. People interested in the research are advised to contact the author for the final version of the publication, or visit the $\mathrm{DOI}$ to the publisher's website.

- The final author version and the galley proof are versions of the publication after peer review.

- The final published version features the final layout of the paper including the volume, issue and page numbers.

Link to publication

\section{General rights}

Copyright and moral rights for the publications made accessible in the public portal are retained by the authors and/or other copyright owners and it is a condition of accessing publications that users recognise and abide by the legal requirements associated with these rights.

- Users may download and print one copy of any publication from the public portal for the purpose of private study or research.

- You may not further distribute the material or use it for any profit-making activity or commercial gain

- You may freely distribute the URL identifying the publication in the public portal.

If the publication is distributed under the terms of Article 25fa of the Dutch Copyright Act, indicated by the "Taverne" license above, please follow below link for the End User Agreement:

www.tue.nl/taverne

Take down policy

If you believe that this document breaches copyright please contact us at:

openaccess@tue.nl

providing details and we will investigate your claim. 


\title{
$\beta$-plane turbulence in a basin with no-slip boundaries
}

\author{
W. Kramer, M. G. van Buren, H. J. H. Clercx, and G. J. F. van Heijst \\ Fluid Dynamics Laboratory, Department of Physics, Eindhoven University of Technology, \\ P.O. Box 513, $5600 \mathrm{MB}$ Eindhoven, The Netherlands
}

(Received 2 June 2005; accepted 29 November 2005; published online 16 February 2006)

\begin{abstract}
On a domain enclosed by no-slip boundaries, two-dimensional, geostrophic flows have been studied by numerical simulations of the Navier-Stokes equation with the $\beta$-plane approximation at intermediate Reynolds numbers and a range of values for $\beta$. The $\beta$ effect causes a refinement of the flow structures, and the presence of basin modes has been revealed by means of frequency spectra. The presence and apparent stability of basin modes on a domain enclosed by no-slip boundaries is a rather surprising observation, because these modes are solutions of the inviscid flow equations on a bounded domain (with free-slip boundaries). To understand the persistence of these basin modes, the viscous boundary layers near the no-slip walls have been investigated. The mean flow in forced simulations shows a zonal band structure, much unlike the regular Fofonoff-like solution observed when free-slip boundary conditions are used. (C) 2006 American Institute of Physics.
\end{abstract}

[DOI: $10.1063 / 1.2173285]$

\section{INTRODUCTION}

The large-scale flow phenomena in the Earth's atmosphere and oceans are mostly two dimensional (2-D) in nature. This is caused by three reasons: (i) the rotation of the Earth, (ii) the geometrical confinement of the flow to a thin fluid layer with large horizontal dimensions and a small vertical dimension, and (iii) the presence of a temperature or salinity stratification in the oceans or a temperature stratification in the atmosphere. Opposing the behavior of threedimensional turbulent flows, 2-D flows may exhibit the spontaneous formation of large, coherent vortex structures in the case of decaying 2-D turbulent flows, or these flows show a large-scale response induced by a small-scale random forcing. A well-known example of the latter phenomenon is the condensation of energy in the largest accessible modes. In that case a domain-filling dipolar vortex emerges in forced 2-D turbulence in a double periodic domain, as predicted by Kraichnan ${ }^{1}$ and observed in numerical simulations by Hossain $e t a l .{ }^{2}$ and Smith and Yakhot. ${ }^{3}$ The mechanism responsible for these processes in decaying and in forced 2-D turbulence is the inverse energy cascade present in 2-D flows. ${ }^{1,4}$

In describing geographical flows, the dynamics are largely influenced by the Coriolis force induced by the rotation of the Earth. On the globe, the magnitude of the Coriolis force depends on the Earth's geographical latitude. The firstorder correction to describe the change in the Coriolis force as function of the meridional latitude is known as the $\beta$-plane approximation (see Refs. 5-7). One of the most important flow phenomena induced by the $\beta$ effect is the Rossby wave, ${ }^{8}$ present both in the Earth's atmosphere and oceans. This westward traveling wave has a wavelength of about $100 \mathrm{~km}$ in the ocean. In the atmosphere, however, their spatial scales are so large that they cover the globe with only a few wavelengths. The presence of Rossby waves changes the evolution of 2-D turbulence on a $\beta$ plane severely compared to ordinary $2-\mathrm{D}$ turbulence. The inverse energy cas- cade has to compete with Rossby wave formation. Simulations of 2-D turbulence with a $\beta$ effect $^{9}$ on a periodic domain showed that the merger of small-scale vortices to large-scale vortices halts at a certain scale, the Rhines scale. The Rossby waves are visible in the flow field as east-west orientated bands with alternating westward and eastward flow. A wellknown example of such a band structure is found in the atmosphere of the planet Jupiter. ${ }^{10}$ Numerical simulations of turbulence on a rotating sphere by Nozawa and Yoden ${ }^{11}$ show the emergence of a similar zonal band structure.

The presence of continents, demarcating the oceans, legitimates a study of the influence of solid boundaries on geostrophic turbulence. Linked up with Rossby waves, especially in the western regions of the oceans, strong boundary currents exist. A famous example is the Gulf Stream, which is formed in the west of the North Atlantic Ocean. After detaching from the coast near Cape Hatteras in North Carolina, the Gulf Stream flows eastward. By transporting relatively warm water toward European waters, the Gulf Stream is responsible for the mild climate in western Europe.

In the present study we are devoted to two-dimensional, geostrophic turbulence on a finite domain with no-slip boundaries. The research is carried out by performing numerical simulations. The remainder of this paper is organized as follows. In Sec. II, we present some theoretical background relevant to 2-D turbulence on a $\beta$ plane and discuss a geostrophic boundary layer model. In Sec. III we describe briefly how the numerical simulations were performed. Sections IV-VII are dedicated to the results of the numerical simulations. Finally, in Sec. VIII some conclusions will be drawn.

\section{TURBULENCE AND WAVES ON A $\beta$ PLANE}

As noted before, the rotation of the Earth (radius $R$ and rotation speed $\Omega$ ) introduces the Coriolis force into the flow dynamics. For two-dimensional flows the effect of the latitu- 
dinal variation of the Coriolis force can be incorporated by using the Coriolis parameter, $f=2 \Omega \sin \phi$, which gives the planetary background vorticity at latitude $\phi$. A linear approximation of the latitudinal variation in the Coriolis parameter is obtained by expanding the Coriolis parameter in a Taylor series to the first order around a reference latitude $\phi_{0}$ :

$$
f\left(\phi_{0}+\delta \phi\right) \approx 2 \Omega \sin \phi_{0}+\frac{2 \Omega \cos \phi_{0}}{R} R \delta \phi=f_{0}+\beta_{0} y .
$$

The $\beta$-plane approximation (1) consists of a constant background rotation $f_{0}=2 \Omega \sin \phi_{0}$ and a linear correction with gradient $\beta_{0}=2 \Omega \cos \phi_{0} / R$. In the local coordinate system $x$ represents the zonal coordinate and $y$, which is introduced by the relation $y=R \delta \phi$, the meridional coordinate. The $\beta$ effect causes an additional term in the $2-\mathrm{D}$ vorticity equation, i.e.,

$$
\frac{\partial \omega}{\partial t}+u \frac{\partial \omega}{\partial x}+v \frac{\partial \omega}{\partial y}+\beta_{0} v=\nu \nabla^{2} \omega+Q .
$$

Here, $\mathbf{u}(t, \mathbf{x})=(u, v, 0)$ is the 2-D Eulerian velocity with components $u$ and $v$ in the $x$ and $y$ direction, respectively. The vorticity $\omega(t, \mathbf{x})$ is a scalar quantity in 2-D flows and is defined as $\omega=(\boldsymbol{\nabla} \times \mathbf{u}) \cdot \hat{\mathbf{e}}_{z}$, with $\hat{\mathbf{e}}_{z}$ the unit vector in the (local) vertical direction. The right-hand side contains the viscous dissipation with kinematic viscosity $\nu$ and the forcing $Q(t, \mathbf{x})=(\boldsymbol{\nabla} \times \mathbf{F}) \cdot \hat{\mathbf{e}}_{z}$, where $\mathbf{F}(t, \mathbf{x})=\left(F_{x}, F_{y}, 0\right)$ represents an externally applied force on the fluid. Two dimensionless numbers can be found by scaling Eq. (2) with a characteristic length scale $L$ and a characteristic velocity scale $U$. First, the Reynolds number is defined as $\operatorname{Re}=L U / \nu$ and gives the importance of the nonlinear interactions relative to viscous dissipation. Second, the $\beta$ number is given by $\beta=\beta_{0} L^{2} / U$, indicating the importance of the $\beta$ term compared with the nonlinear term. Alternatively, instead of the dimensionless $\beta$ number the $\beta$-plane Rossby number Ro can be used, the relation between both numbers is given by $\mathrm{Ro}=\beta^{-1}$. For details on this topic one can consult, e.g., Pedlosky ${ }^{5}$ or Kundu. ${ }^{6}$ The noninertial force induced by the gradient in the planetary background vorticity is responsible for a special kind of planetary wave, the so-called Rossby waves. ${ }^{5,8}$ The presence of the mechanism leading to Rossby waves alters the dynamics and evolution of 2-D turbulent flows drastically. A characteristic feature of 2-D turbulence is the inverse energy cascade, i.e., the kinetic energy transfer from small scales to the larger scales of the flow. This eventually results in an increasing size of the energy-containing eddies (most prominently visible in decaying 2-D turbulence; see, for example, Matthaeus and Montgomery ${ }^{12}$ and McWilliams ${ }^{13}$ ). With the presence of the $\beta$ effect, however, the eddies do not grow in size unlimitedly. The $\beta$ effect becomes increasingly important in the dynamics of the flow as the vortices grow in time (and their characteristic size compares with the typical length scale over which appreciable changes of the planetary background rotation are felt). At a certain scale the inverse energy cascade, which is a result of the nonlinear term in (2), has to compete with the mechanism promoting Rossby waves, and the character of the flow changes from turbulent to wave-like motion. This scale where the inverse energy cascade is halted is the so-called Rhines scale, ${ }^{9}$

$$
k_{\beta}^{-1}=\sqrt{\frac{2 U}{\beta_{0}}},
$$

which is a transition scale between the turbulent $\left(k \gtrsim k_{\beta}\right)$ and the Rossby-wave $\left(k \leq k_{\beta}\right)$ regime.

The Rossby wave can be described mathematically by solving the linearized, inviscid vorticity equation (2) for the streamfunction $\psi(t, \mathbf{x})$ (defined as $u=-\partial \psi / \partial y, v=\partial \psi / \partial x$ ), yielding

$$
\frac{\partial}{\partial t} \nabla^{2} \psi+\beta_{0} \frac{\partial \psi}{\partial x}=0 .
$$

The solution can be expressed by any superposition of plane waves, $\psi(t, \mathbf{x})=\cos (\mathbf{k} \cdot \mathbf{x}-\sigma t)$, with the frequency $\sigma$ obeying the dispersion relation

$$
\sigma=\frac{-\beta_{0} k}{k^{2}+l^{2}} \text {. }
$$

Here, $k$ and $l$ denote the wave numbers in zonal and meridional direction, respectively. The asymmetry in the dispersion relation is believed to be a reason behind the occurrence of highly anisotropic, zonal jets in forced turbulence simulations on a periodic domain (see, for example, the review by Rhines ${ }^{14}$ ) and turbulence simulations on a rotating sphere. $^{11,15}$

The confinement of the flow by boundaries changes the dynamics of the Rossby waves completely, as zonal jets are obstructed by the meridional walls. On a rectangular domain enclosed by impenetrable free-slip boundaries, a special type of solution to (4) exists, known as the basin mode. On a square domain with size $L_{\mathcal{D}}$, the basin mode reads ${ }^{5}$ as

$$
\begin{aligned}
& \psi_{n m}(t, \mathbf{x})=\cos \left(\sigma_{n m} t+\frac{\beta_{0} x}{2 \sigma_{n m}}\right) \sin \left(\frac{n \pi x}{L_{\mathcal{D}}}\right) \sin \left(\frac{m \pi y}{L_{\mathcal{D}}}\right), \\
& n, m=1,2, \ldots,
\end{aligned}
$$

with the dispersion relation

$$
\sigma_{n m}=-\frac{\beta_{0} L_{\mathcal{D}}}{2 \pi \sqrt{n^{2}+m^{2}}} .
$$

The "wave numbers" $n$ and $m$ cannot directly be related to the real length scales in the flow, because of the specific construction of (6). However, each basin mode can be written as a combination of four Rossby waves, $\left(k_{i}, l_{j}\right)_{i, j=1,2}$ with $k_{i}$ and $l_{j}$ given by ${ }^{16}$

$$
\begin{array}{ll}
k_{1}=\frac{\pi}{L_{\mathcal{D}}}\left(\sqrt{n^{2}+m^{2}}-n\right), & l_{1}=-\frac{m \pi}{L_{\mathcal{D}}}, \\
k_{2}=\frac{\pi}{L_{\mathcal{D}}}\left(\sqrt{n^{2}+m^{2}}+n\right), & l_{2}=\frac{m \pi}{L_{\mathcal{D}}} .
\end{array}
$$

In order to find an average wave number, we calculate the root-mean-square (rms) magnitude of the four Rossby wave vectors 


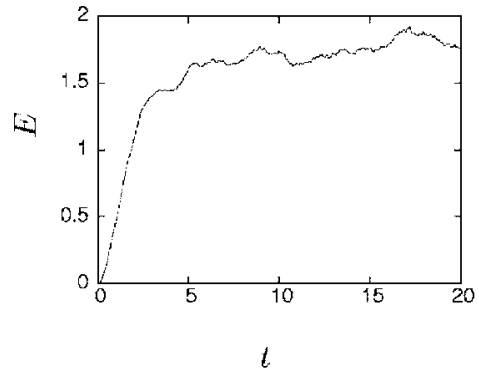

(a) energy

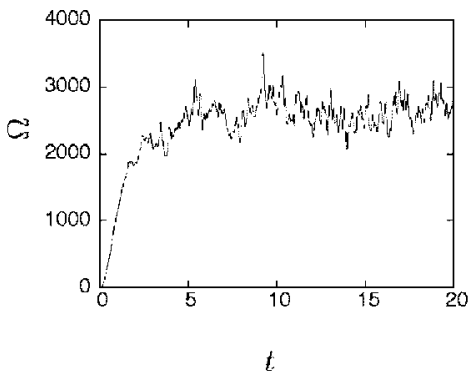

(b) enstrophy

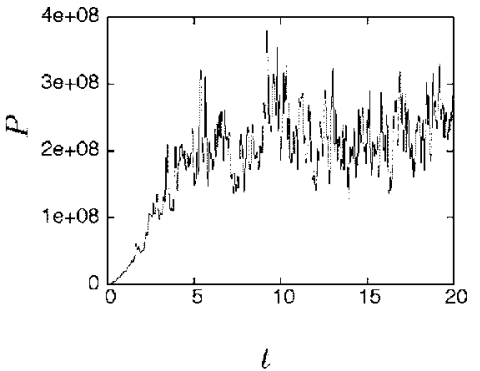

(c) palinstrophy

FIG. 1. The time evolution of the total kinetic energy $E$ (a), the enstrophy $\Omega$ (b), and the palinstrophy $P$ (c) for $\beta_{0}=800$ and $\nu^{\prime}=10^{-4}$. A quasisteady state is reached at $t \approx 10$; after this time the global quantities show a fluctuating behavior, but there is no net increase.

$$
|\mathbf{K}|=\frac{\sqrt{2} \pi}{L_{\mathcal{D}}}\left(n^{2}+m^{2}\right)^{1 / 2} .
$$

By means of (9), the basin-mode frequency (7) can be approached by

$$
\sigma=-\frac{\beta_{0}}{\sqrt{2}|\mathbf{K}|} .
$$

We need to stress that for the derivation of basin modes inviscid conditions are assumed, including the associated free-slip boundary condition. A point of interest is how the basin modes behave in a domain enclosed by no-slip boundaries. Besides, one of the topics to be considered is a scaling theory for the viscous boundary layer formed on the no-slip boundary. This will be discussed in Sec. VII.

The influence of no-slip boundary conditions is not limited to the boundary layer itself, as boundary layers detach from the wall and are injected into the flow as vorticity filaments. This source of small-scale vorticity interferes with the inverse energy cascade to larger scales. ${ }^{17}$ Hence, the investigation of the influence of the no-slip boundaries on forced $\beta$-plane turbulence seems interesting and useful. Forced $\beta$-plane turbulence enclosed by free-slip boundaries was recently studied by LaCasce. ${ }^{18}$ His work confirms the presence of basin modes and studies their influence on the isotropy of the turbulence. In this paper we touch on both these topics, in light of a more general look at the flow structures.

\section{NUMERICAL MODEL}

The flow simulations have been executed with a Chebyshev pseudospectral solver, developed by Clercx. ${ }^{19}$ The use of Chebyshev polynomials for the expansion of the flow variables allows us to apply nonperiodic boundary conditions. The computational domain $\mathcal{D}$ consists of the square $[-1,1] \times[-1,1]$. At the boundaries of this domain, no-slip conditions are imposed, yielding the fact that both the normal and tangential velocity components must vanish at the boundaries. The flow properties on the domain are calculated on a Gauss-Lobatto grid. The density of the Gauss-Lobatto grid points increases toward the boundaries, which enables us to study boundary layers in more detail. The evolution of (2) is performed in spectral space by evolving the spectral coefficients of the velocity and vorticity in time. Time step- ping is based on the second-order-accurate Adams-Bashforth Crank-Nicolson scheme. On behalf of the efficiency, the calculation of the nonlinear term takes place in physical space. ${ }^{20}$ The $\beta$ effect is simply added to the nonlinear term by computing the product $v(\partial \omega / \partial y+\beta)$ in physical space.

Like in nature (e.g., the wind stress on the surface waves), the forcing in our numerical simulations is applied on a certain scale. In spectral space this can be realized by allowing only wave vectors $\mathbf{k}$ with length $a \leqslant|\mathbf{k}| \leqslant b$,

$$
Q(t, \mathbf{x})=\sum_{a \leqslant|\mathbf{k}| \leqslant b} \hat{q}(t,|\mathbf{k}|) e^{i \mathbf{k} \cdot \mathbf{x}} .
$$

For the sake of homogeneous forcing in time, a complex random phase factor is included in $\hat{q}(t,|\mathbf{k}|)$. The forcing is based upon a first-order Markov process, which incorporates a correlation time $\tau$. For small $\tau$ the forcing is nearly random in time, while $\tau \rightarrow \infty$ results in static-in-time forcing. This kind of forcing was first used in numerical simulations by Lilly $^{21}$ and later in Ref. 22.

The simulations have been executed with a resolution of $257 \times 257$ Chebyshev expansion coefficients and a time step $\Delta t=10^{-4}$. It should be noted, however, that the nonlinear contribution is computed with a resolution of $385 \times 385$ Chebyshev expansion coefficients in order to dealiase the computation. With this resolution and time step the simulations are well resolved and stable. For the simulations we have to set a priori a prescribed value to the dimensionless viscosity $\nu^{\prime}$ and the dimensionless gradient in the background vorticity $\beta_{0}^{\prime}$. The actual value of the Reynolds number and the $\beta$ number can be calculated once the rms velocity is known. For most simulations the viscosity parameter was set to $\nu^{\prime}=10^{-4}$ and $\beta_{0}^{\prime}$ is chosen in the range $[0,800]$. The forcing was applied in the range $14 \pi \leqslant|\mathbf{k}| \leqslant 16 \pi$, so that in the present simulations the forcing scale is always smaller than the Rhines scale. The forcing correlation time is set to $\tau=1$, which gives a relatively slowly varying forcing field. As an initial condition a zero velocity field is used. All simulations have been run until the total kinetic energy $E=\frac{1}{2} \int_{\mathcal{D}} \mathbf{u}^{2} d A$, the enstrophy $\Omega=\frac{1}{2} \int_{\mathcal{D}} \omega^{2} d A$, and the palinstrophy $P=\frac{1}{2} \int_{\mathcal{D}}|\nabla \omega|^{2} d A$, reached their respective, final equilibrium values (see Fig. 1). After this quasisteady state was reached, at the dimensionless time $t \approx 10$, the simulation was continued for an additional period of time in order to obtain reliable results that are statistically stationary. (In this paper, 
TABLE I. Several quantities for the forced simulations with varying $\beta_{0}^{\prime}$ : the time-averaged rms velocity $U_{\text {rms }}$ during the quasisteady state, the Rhines wave number $k_{\beta}(3)$, the anisotropy $\mathcal{A}(14)$, and the vorticity kurtosis $K_{\omega}$ (13). The boundary layers, with the extreme vorticity values, are not included in the calculation of $K_{\omega}$. The standard deviation for the energy is given in the table, the error margin is about 0.2 for $K_{\omega}$, and the data for $\mathcal{A}$ indicate (within the error margin) isotropy of the turbulent flow. Data for $U_{\mathrm{rms}}$ is not available for $\beta_{0}=600$; the value of $k_{\beta}$ is estimated for this case.

\begin{tabular}{lllll}
\hline \hline$\beta$ & $U_{\text {rms }}$ & $k_{\beta}$ & $\mathcal{A}$ & $K_{\omega}$ \\
\hline 0 & $1.67 \pm 0.15$ & 0 & 0.08 & 7.8 \\
100 & $1.16 \pm 0.06$ & 6.6 & -0.04 & 3.7 \\
200 & $1.08 \pm 0.03$ & 9.6 & -0.04 & 3.2 \\
400 & $0.94 \pm 0.02$ & 15 & -0.08 & 2.9 \\
600 & - & 19 & 0.04 & 3.0 \\
800 & $0.78 \pm 0.03$ & 23 & 0.05 & 2.9 \\
\hline \hline
\end{tabular}

these statistically stationary states are also referred to as quasisteady states.) The forcing amplitude is set to such a value that the rms velocity averaged over time during the quasisteady state is of order one. If we compute the Reynolds number and the $\beta$ number with the rms velocity and the half-width of the domain, this results in $\operatorname{Re} \approx 10000$ and $\beta \approx \beta_{0}^{\prime}$ for the quasisteady state. Generally, we are only interested in observing trends in the flow for varying values of $\beta$, and therefore we will assume $\beta=\beta_{0}^{\prime}$. The time-averaged rms values of the velocity during the quasisteady state are given in Table I. The Reynolds number is substantially smaller than commonly used in 2-D periodic turbulence simulations, and is due to the restricted number of Chebyshev modes that can be used in a feasible direct numerical simulation of turbulence on a 2-D domain with no-slip boundaries (that automatically requires the use of a NavierStokes solver, thus also excluding the use of hyperviscosity operators). For reference, a basin with a width of $4000 \mathrm{~km}$ at a moderate latitude of $\phi_{0}=45^{\circ}$ and typical velocity of $0.2 \mathrm{~m} / \mathrm{s}$ would correspond to a $\beta$ number of $\beta \approx 300$.

\section{GENERAL FLOW PHENOMENA}

In order to obtain a general impression of the influence of the $\beta$ effect on the flow, we present some snapshots of typical flow fields with different values for $\beta$. In Figs. 2(a)-2(c), contour plots of the vorticity are shown, while Figs. 2(d)-2(f) depict the corresponding streamline patterns. In the simulation without a $\beta$ effect [panels (a) and (d)], a large counterclockwise rotating vortex has emerged, filling the majority of the flow domain. Obviously, this is a result of the inverse energy cascade. The formation of such a single vortex on a square no-slip domain, a process known as spontaneous spin-up, has been studied in more detail by Clercx et $a l .{ }^{23,24}$ for the case of decaying turbulence. Spin-up on a square no-slip domain in forced turbulence was, among others, studied by Molenaar et al., ${ }^{25}$ where emphasis has been given to the role of flow-boundary interactions.

As can be observed in Figs. 2(e) and 2(f), the $\beta$ plane suppresses the formation of one large vortex. This can be explained as follows. The scales of the flow structures grow due to the inverse energy cascade. As the Rhines scale is reached, the $\beta$ effect becomes more important than the advection process and the inverse cascade comes to a halt. It can be observed that a larger value of $\beta$ leads to further refinement of the present flow scales, which is in agreement with (3).

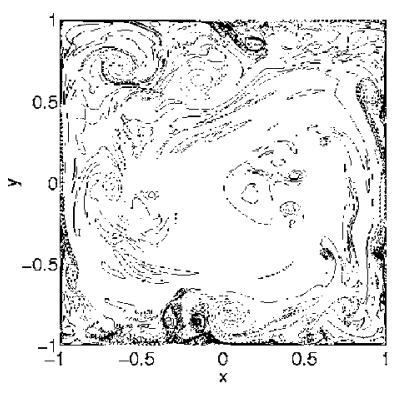

(a) vorticity, $\beta \quad 0$

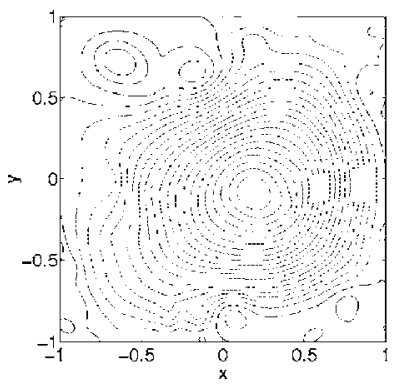

(d) stream function, $\beta=0$

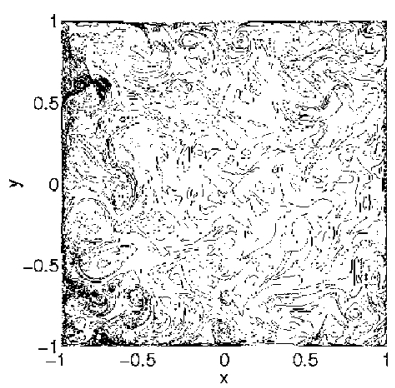

(b) vorticity, $\beta \quad 100$

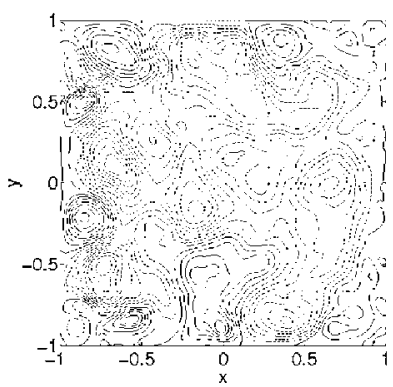

(e) stream function, $\beta=100$

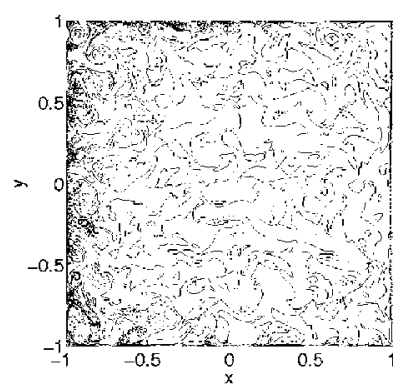

(c) vorticity, $\beta \quad 400$

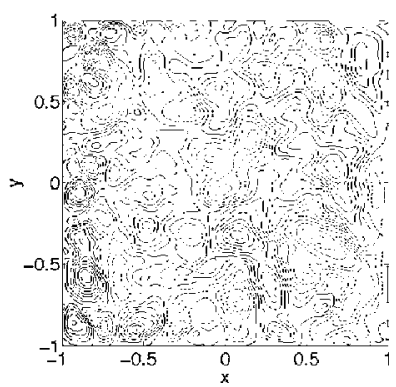

FIG. 2. Vorticity and streamfunction on a domain with no-slip boundaries. For these simulations $\operatorname{Re} \approx 10000$. The upper panels show the vorticity and the lower panels the streamfunction for (a), (d) $\beta=0$, (b), (e) $\beta=100$, and (c), (f) $\beta=400$. The solid lines represent positive values and the dashed lines represent negative values. 


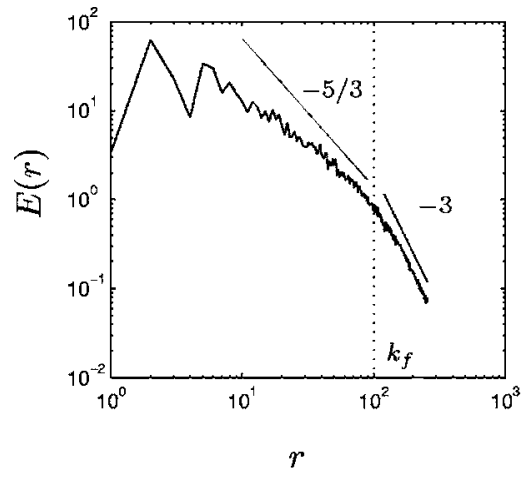

(a) $\beta=0$

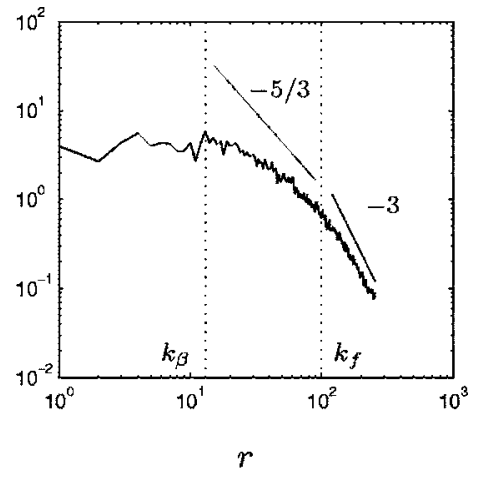

(b) $\beta=100$

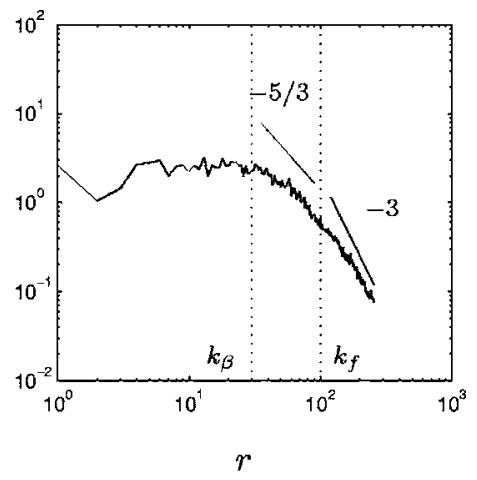

(c) $\beta=400$

FIG. 3. Chebyshev spectra of the kinetic energy $E(r)$ for $\operatorname{Re} \approx 10000$ and $\beta=0$ (a), 100 (b), and 400 (c). The 1-D spectrum is calculated by summing over the Chebyshev coeffients of the energy $\left|E_{p, q}\right|$ for constant $r=\left(p^{2}+q^{2}\right)^{1 / 2}$. The spectrum is averaged over 11 realizations of the flow field, equally distributed in the time period $t=[10,20]$. The approximate locations of the forcing wave number $k_{f}$ and Rhines wave number $k_{\beta}$ are also specified (see the text for details). The drawn lines represent the classical power law scalings of $k^{-5 / 3}$ and $k^{-3}$ corresponding to the inverse energy cascade and enstrophy cascade, respectively.

To complement these observations, one-dimensional Chebyshev spectra of the energy are examined (see Fig. 3). Note that the usual Fourier spectra are not applicable on a nonperiodic domain and there is no one-to-one correspondence between Fourier and Chebyshev spectra. For instance, the applied forcing, which is localized in the Fourier spectrum at $14 \pi \leqslant|\mathbf{k}| \leqslant 16 \pi$, has a significant contribution to the Chebyshev spectrum for all modes $n \lesssim 100$. However, a $k^{-\alpha}$ Fourier spectrum corresponds to the same power law in a Chebyshev spectrum. ${ }^{17}$ The spectrum for $\beta=0$ reveals a slope slightly less than $-5 / 3$ for the modes smaller than the forcing wave number, supporting the presence of an inverse energy cascade. For the modes $n \gtrsim 100$ a short -3 slope is present, which can be attributed to the enstrophy cascade. Comparing the spectra reveals that the spectrum becomes flat for scales smaller than the Rhines wave number. This observation is in agreement with an obstruction of the inverse energy cascade by the $\beta$ effect. A strong collapse of the spectrum for scales smaller than the Rhines scale, as found by the authors of Ref. 22 on a periodic domain, cannot be observed. This is due to the characteristics of the Chebyshev spectrum; it is strongly aliased at lower modes, as explained earlier. The increase in $\beta$ number results in a spectrum that remains approximately flat for a larger span of Chebyshev modes, as supported by a larger Rhines wave number. For modes larger than the forcing wave number, an approximate slope -3 remains visible, as these modes are unaffected by the $\beta$ effect.

The demand that the velocity must vanish at the no-slip boundary causes high-velocity gradients, which is expressed by high-vorticity values near the boundaries. By means of advection, the boundary layers roll up and detach, causing the thin filaments of high vorticity in the interior of the flow, clearly present in the vorticity field for $\beta=0$ [Fig. 2(a)]. These interactions of the flow with the boundaries are only visible in the western part of the domain for $\beta=100$ and 400 . The drift of vorticity to the west, due to the presence of basin modes, makes an interaction of vortex structures with the eastern boundary unlikely. Vorticity is accumulated against the western boundary, which is visible in the left parts of
Figs. 2(b) and 2(c). The topic of detaching boundary layers will receive more attention in the next section, where the dynamics of basin modes in square domains with no-slip boundaries is discussed in more detail.

Similar to the vorticity accumulation, an intensification of energy at the western boundary is visualized by a locally denser streamline pattern in the left regions of Figs. 2(e) and 2(f). In fact, this appears to be an inertial boundary layer based on the reflection of Rossby waves by the western impermeable boundary. In the presence of a westward mean flow $U$, it follows that Rossby waves with scales smaller than $^{5}$

$$
\delta_{I} \approx 2 \pi \sqrt{\frac{U}{\beta_{0}}}
$$

are trapped at the western boundary. As time proceeds, energy-containing vortices with size smaller than (12) accumulate in the western part of the domain and form a highly energetic boundary layer. In Fig. 4 the meridionally integrated energy density, $e(x)=\frac{1}{2} \int_{-1}^{+1}|\mathbf{u}|^{2} d y$, is plotted as a function of the zonal coordinate $x$ for four simulations with different values of $\beta$. In general, a substantial part of the kinetic energy of the flow is located within the theoretical inertial boundary layer (12), represented by the dotted lines. Note that the no-slip boundary conditions do not affect the inertial boundary layer; viscous effects are limited to very thin viscous boundary layers with thickness $\delta_{v}$, and $\delta_{\nu} / \delta_{I} \approx \sqrt{\beta / \operatorname{Re}} \ll 1$.

The suppression of the formation of large-scale vortices by the $\beta$ effect is confirmed by the kurtosis $K_{\omega}$ of the vorticity. This quantity is defined as

$$
K_{\omega}=\frac{\int_{\mathcal{D}} \omega^{4} d A \int_{\mathcal{D}} d A}{\left(\int_{\mathcal{D}} \omega^{2} d A\right)^{2}} .
$$

The results for our numerical simulations are listed in Table I. Throughout each run, the kurtosis and anisotropy (see be- 


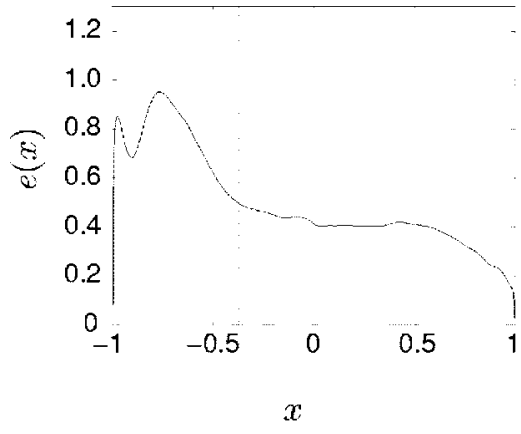

(a) $\beta-100$

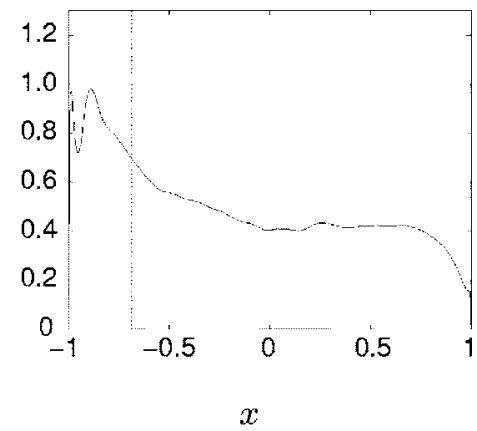

(b) $\beta-400$

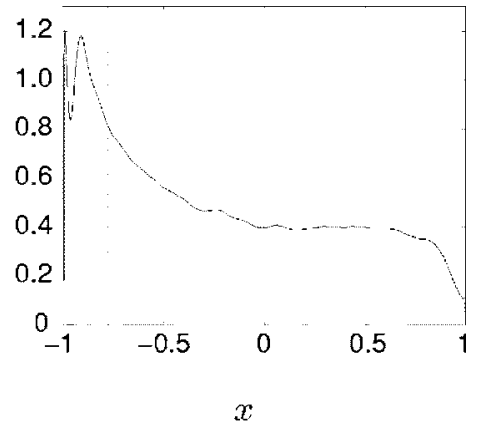

(c) $\beta-800$

FIG. 4. Meridionally integrated energy $e(x)=\frac{1}{2} \int_{-1}^{+1}|\mathbf{u}|^{2} d y$ as function of the zonal coordinate for (a) $\beta=100$, (b) 400 , and (c) 800 . In these simulations $\operatorname{Re} \approx 10000$. The vertical, dotted lines indicate the inertial boundary layer thickness $\delta_{I}$ as specified by (12).

low) values have been evaluated at five different times and averaged. For $\beta=0$, the presence of some large vortices and strong vorticity filaments result in a high value for the kurtosis [see Fig. 2(a)]. Including the $\beta$ effect makes the kurtosis decrease rapidly to the Gaussian value of $K_{\omega}=3$, indicating the absence of coherent vortices and vorticity filaments. The flow on a $\beta$ plane is actually dominated by the presence of basin modes, as described in the next section.

The anisotropy $\mathcal{A}$ of the flow has been determined by computing $^{22}$

$$
\mathcal{A}=\frac{\int_{\mathcal{D}}\left(u^{2}-v^{2}\right) d A}{\int_{\mathcal{D}}\left(u^{2}+v^{2}\right) d A} .
$$

For $\beta=0$, a rather isotropic flow has developed, as indeed could be expected. However, as far as our numerical simulations are concerned, the $\beta$ effect seems not to induce any appreciable anisotropy in the turbulent flow field, as can be learned from Table I. As (14) gives an integral measure of the isotropy, it is also useful to investigate the twodimensional energy spectra of the flow (see Fig. 5). The spectrum $\beta=0$ is isotropic, and the spectrum for $\beta=100$ is indistinguishable from the isotropic case. For $\beta=400$ the spectrum becomes slightly anisotropic, with relatively more energy located in Chebyshev modes $T_{p, q}$ above the line $p=q$. These modes correspond to structures with their largest scale along the $x$ axis, i.e., the zonal direction. The lack of a (strong) anisotropy is purely due to the confinement of the flow that reduces the emergence of a clear band structure, as simulations on a periodic domain show that the flow becomes significantly more anisotropic for increasing $\beta{ }^{9}$ LaCasce has shown that the flow field on the square bounded domain with free-slip boundaries is also nearly isotropic. ${ }^{18}$ However, we also need to reemphasize his remarks on the possible influence of the (isotropic) forcing and the aspect ratio of the domain on the formation of an isotropic flow field.

\section{BASIN MODES IN A SQUARE DOMAIN WITH NO-SLIP WALLS}

Although the snapshots of the flow (Fig. 2) do not show organized flow structures, the presence of basin modes is profoundly revealed by the frequency spectra shown in Fig. 6 . These spectra were obtained by evaluating the line integral over the streamfunction, extending from the domain center $(0,0)$ to the upper right corner $(1,1)$ as a function of time. In Fig. 6 the frequency spectra of four runs with different values for $\beta$ are compared. The position of the observed frequency peaks coincide exactly with the theoretical basin mode frequencies (7), clearly revealing the existence of basin modes in a square domain with no-slip walls, a rather surprising observation. Comparing the frequency spectra, it can be observed that increasing the $\beta$ number leads to the excitation of finer basin modes. This phenomenon can be explained by the Rhines scale (3). For small $\beta$ numbers (a large

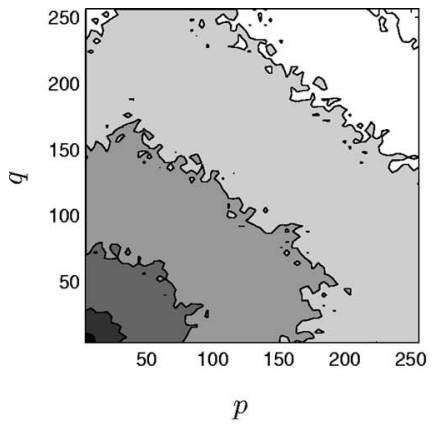

(a) $\beta=0$

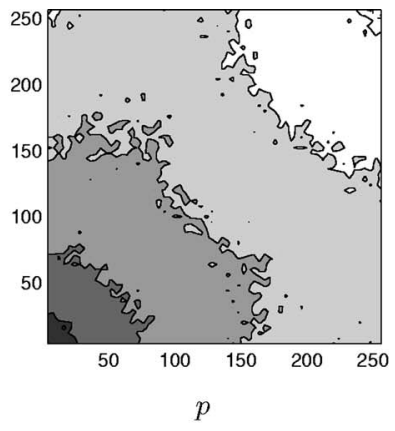

(b) $\beta=100$

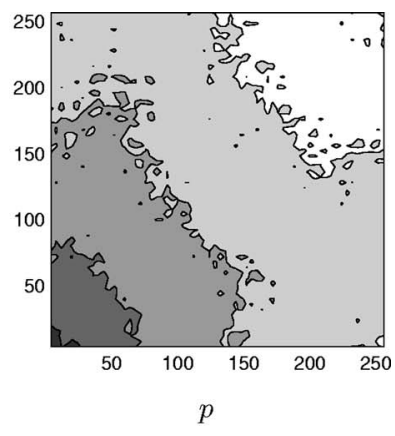

(c) $\beta=400$
FIG. 5. 2-D Chebyshev spectra of the kinetic energy for $\operatorname{Re} \approx 10000$ and $\beta$ $=0(\mathrm{a}), 100(\mathrm{~b})$, and $400(\mathrm{c})$. Along the axis are given the mode $p$ for the zonal and $q$ for the meridional direction. The spectrum is averaged over 11 realizations of the flow field, equally distributed in the time period $t=[10,20]$. Plotted are the contours of $\log ^{10}\left(\left|E_{p, q}\right|\right)$ for contour levels $(0,-1, \ldots,-5)$, where $E_{p, q}$ are the Chebyshev coefficients of the energy. 
$(1,3)$

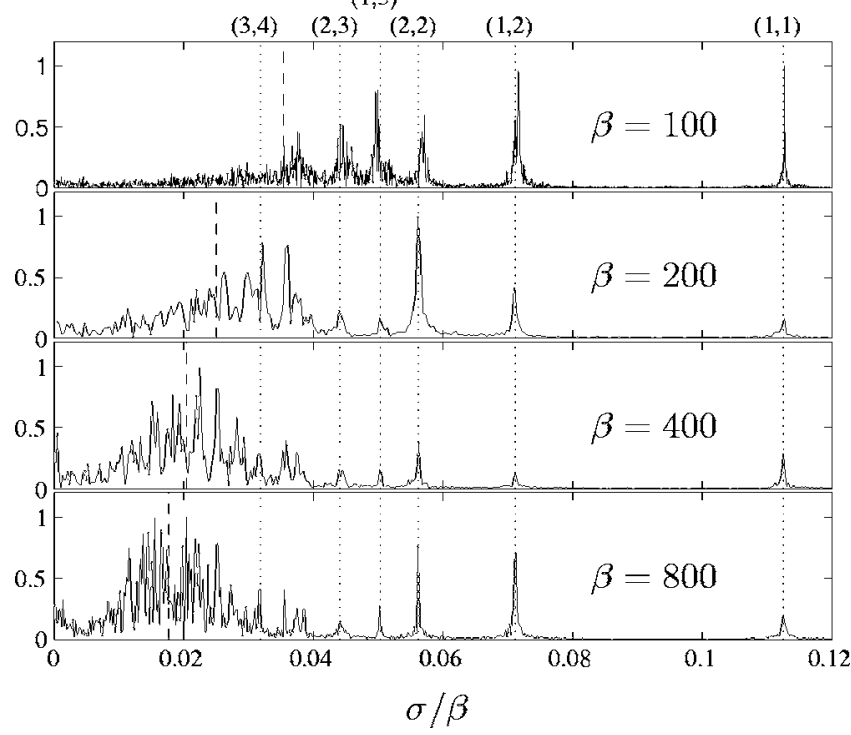

FIG. 6. Frequency spectra for $\beta=100,200,400$, and 800 . The frequency $\sigma$ has been scaled with $\beta$, such that for any value of $\beta$ the frequency of a certain basin mode has the same position. The vertical axis is normalized with respect to the highest peak. The dotted lines represent some of the basin mode frequencies according to (7). The dashed lines represent the frequencies belonging to the Rhines scale, given by (15). For all simulations $\operatorname{Re} \approx 10000$.

Rhines scale) the inverse energy cascade is less impeded and the flow structures tend to larger scales, thus graver basin modes. The other way around, a large $\beta$ yields a small Rhines scale, resulting in finer basin modes. An estimate can be given by substituting the Rhines wave number $k_{\beta}=\sqrt{\beta_{0} / 2 U}$ into the dispersion relation (10), leading to a Rhines-scale related cutoff frequency of

$$
\sigma_{\beta}=\sqrt{\beta_{0} U}
$$

In each spectrum this frequency is plotted with a dashed line, indicating the frequency regime where a rather gradual transition between the Rossby wave and turbulence regimes occurs. These observations are in agreement with an arrest of the inverse energy cascade at the Rhines wave number, as found in the 1-D energy spectra (Fig. 3). At this point the energy transferred to larger scales by the inverse cascade is then used to excite basin modes with wavelengths around the Rhines scale. Therefore, the resulting flow field consists mainly of a superposition of basin modes. Taking another look at the streamfunction in Fig. 2, we can conclude that the observed structures are due to this superposition of basin modes. On top of the basin modes, there are some smallscale structures that are mainly produced by the interaction between the flow and the viscous boundary layers. Most of these small-scale structures are too weak to be seen in the streamfunction plot, but are clearly visible in the vorticity plots.

It should be noticed that, since the domain is a square, a frequency degeneration exists for the basin modes. That is, the basin modes $(n, m)$ and $(m, n)$ occupy the same frequency, i.e., $\sigma_{n m}=\sigma_{m n}$. Due to this frequency degeneration, one is unable to tell whether, e.g., the second largest fre- quency peak belongs to the $(1,2)$ mode or the $(2,1)$ mode (or both). (On behalf of simplicity, only one mode is denoted in Fig. 6.) Because the basin modes play such an essential role in the simulations, they have been studied individually. Several individual basin modes were imposed as an initial condition and, subsequently, left to decay. Since the external forcing would have disturbed the shape of the basin mode, no forcing was applied for this particular case. Although the basin modes do not comply with the no-slip boundary conditions, they have been found to be remarkably stable, i.e., the general flow structure is maintained for tens of cycles and the propagation speed has been found to be in agreement with the theory. In order to get an impression, we present some snapshots of several developed basin modes on a noslip domain in Fig. 7. It should be emphasized that at this moment these basin modes have completed already tens of cycles.

A reason why the single basin modes are stable on a no-slip domain is believed to be related to the behavior of the thin viscous boundary layers. (The thickness of the viscous boundary layer will be discussed in more detail in Sec. VII.) The velocity component parallel to the boundary is reduced to zero in the viscous boundary layer, as is required to satisfy the no-slip condition. Just outside the boundary layer this velocity remains unaltered and is nearly equal to the velocity found when applying free-slip boundary conditions. Hence, the outer side of the viscous boundary layer effectively provides a condition similar to the free-slip boundary condition, which is used for the derivation of basin modes. For large Reynolds numbers the viscous boundary layers are thin, thus the effective area for the basin modes is practically equal to the container area. However, boundary layers might detach from the wall, thus affecting the flow in the interior of the domain. As the velocity just outside the boundary layer is alternating in time due to the Rossby wave propagation, the vorticity in the boundary layer is continuingly changing sign in time. Therefore, the period the boundary layer exists might be too short to detach from the wall in the form of high-amplitude vorticity filaments that eventually might roll up to form stability of the basin modes does not only depend on the Reynolds number, but also on the $\beta$ number. For example, if $\beta=25$ the basin mode $(1,1)$ is not stable, even for $\operatorname{Re}=10000$. The frequency of the basin mode is lower for smaller $\beta$ numbers. On the other hand, the advection time scale related to the detachment of the boundary layer is supposed to be fixed, as the shape of the basin modes remains the same for different $\beta$ 's. Hence, there is more time for the boundary layers to detach from the wall if $\beta$ is smaller. The same can be said for the higher-order modes; frequencies for these modes are smaller for increasing order of the mode for a fixed $\beta$. Therefore, higher-order basin modes are less stable. Of course, these findings are related to the fact that higher-order basin modes are present in the turbulence simulations if $\beta$ is sufficiently large (see Fig. 6). A more elaborate stability study for single basin modes could give more insight into the exact relation. For now we conclude that the influence of the no-slip boundary condition is rather restricted, as the viscous boundary layer is stable provided $\beta$ is large enough. 

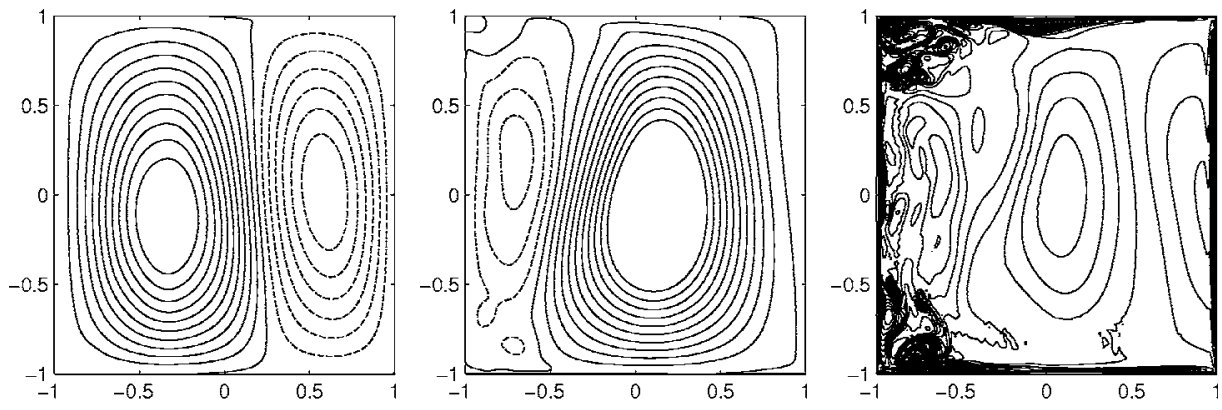

(a) $\psi(t, \mathrm{x}), \beta=50$

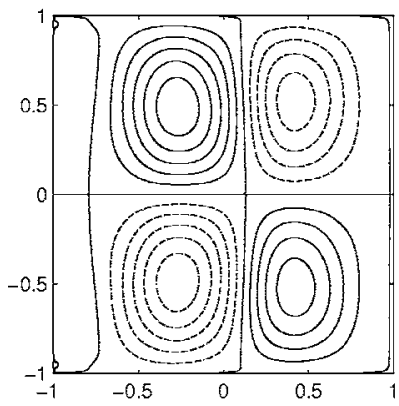

(d) $(1,2), \beta-200$ (b) $\psi(t, \mathrm{x}), \beta=25$

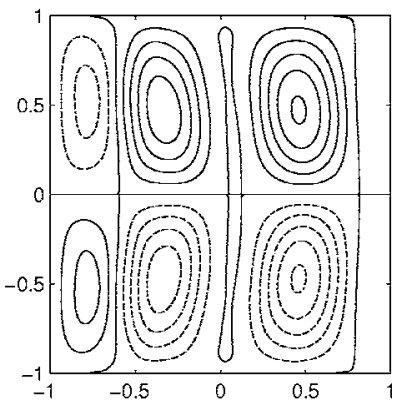

(e) $(2,2), \beta-200$

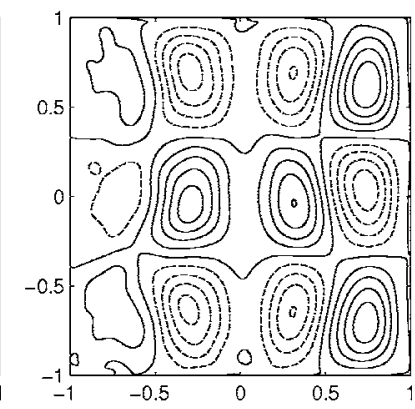

(f) $(2,3), \beta-200$
FIG. 7. Simulations of individual basin modes on a domain with no-slip boundaries for an initial Reynolds number of $\operatorname{Re}=4000$. The lowest-order basin mode $(1,1)$ is stable for $\beta>50$ (a), but unstable for $\beta=25$ (b)-(c). The lower panels (d) - (f) show several higher-order basin modes for $\beta=200$.

\section{WEAK BAND STRUCTURES IN TIME-MEAN FLOWS}

The westward propagating basin modes often drive a mean flow, which consists of two recirculation cells: one attached to the northern boundary and another attached to the southern boundary. Such a mean flow resembles the solution found by Fofonoff for the steady unforced inviscid flow on a square domain mimicking a frictionless homogeneous ocean. ${ }^{26}$ The solution found by Fofonoff consists of an anticyclonic gyre filling the northern half of the domain and a cyclonic gyre the southern half. For steady inviscid flows a functional relation exists between the mean streamfunction $\bar{\psi}$ and the mean potential vorticity, viz., $\bar{q}=\bar{\omega}+\beta_{0} y$, i.e., the Jacobian $J(\bar{\psi}, \bar{q})$ is zero. Fofonoff chose a linear relation, yielding his two-gyre solution, but any functional relation satisfies $J(\bar{\psi}, \bar{q})=0$ and would, in principle, suffice. Additional support for the existence of the Fofonoff cells is, how- ever, provided by equilibrium statistical mechanics for inviscid bounded geostrophic flows (with free-slip boundary conditions) that also predicts this linear relation between the mean potential vorticity and the mean streamfunction. ${ }^{27}$

The time-averaged streamfunction for several of our simulations are given in Fig. 8. The kinetic energy associated with the mean flow is about $2 \%-4 \%$ of the total kinetic energy. Remarkable is the appearance of a band structure consisting of circulation cells elongated in the zonal direction that resembles the zonal band structure on a periodic domain. ${ }^{9}$ The number of bands is increasing with the $\beta$ number, i.e., the width of the bands is prescribed by the Rhines scale. The zonal band structure on a periodic domain is due to the presence of Rossby waves with meridional wave numbers close to the Rhines wave number and a zonal wave number equal to zero. On a bounded domain, there is no basin mode that resembles the zonal band structure found for
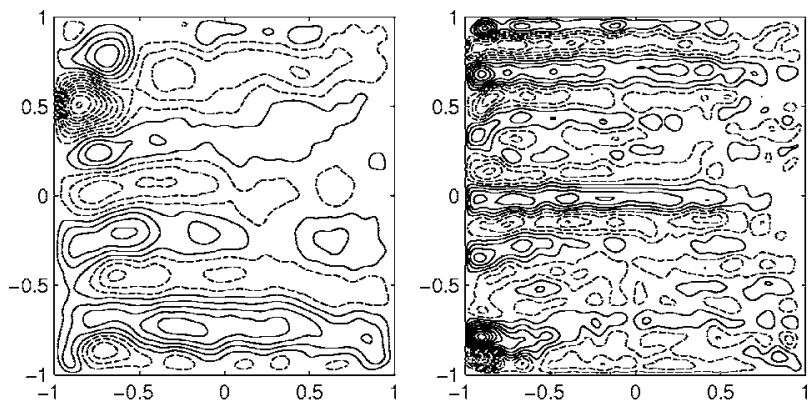

(a) $\beta-100$ (b) $\beta-400$

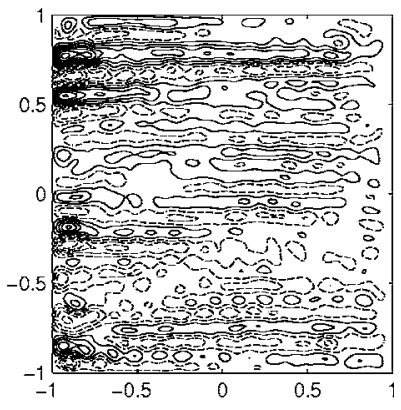

(c) $\beta-800$
FIG. 8. Contour plot of the timeaveraged streamfunction for (a) $\beta$ $=100$, (b) 400, and (c) 800. Contour lines are drawn for positive values of the streamfunction and dashed for negative values. 


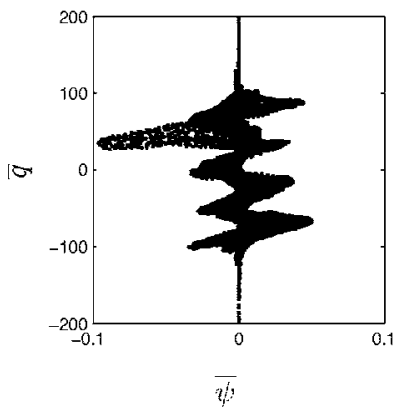

(a) $\bar{q}(\bar{\psi})$, entire domain

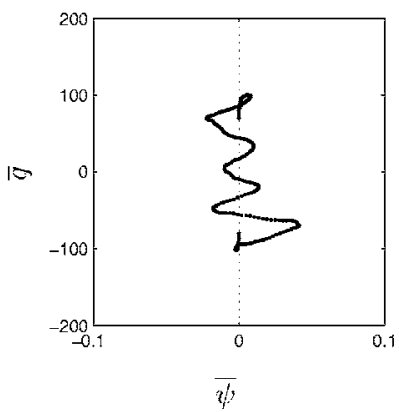

(b) $\bar{q}(\bar{\phi}), x-0$

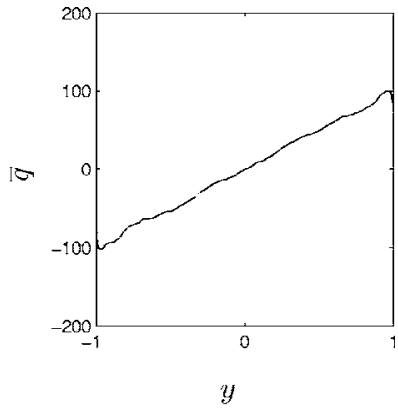

(c) $\bar{q}(y), x \quad 0$
FIG. 9. Scatter plot of the mean potential vorticity $\bar{q}=\bar{\omega}+\beta_{0} y$ versus the mean streamfunction $\bar{\psi}$ (a) and the scatter plot for the line $x=0(\mathrm{~b})$, and the mean potential vorticity versus the latitude $y$ for the line $x=0$ (c). For all cases $\beta=100$. Time averaging is done over 2500 flow fields during the quasisteady state $(t=[10,20])$. the mean flow. The propagating part of the basin mode (6) prevents such a zonal domain-sized band in the solution, even for basin modes with $n=1$. Furthermore, basin modes itself do not induce any mean flow, as the propagating part vanishes when averaged in time. Like the Fofonoff solution, inertial effects need to be considered in addition to the $\beta$ term to get a nonzero mean flow. The streamline pattern is denser in the western part of the domain, where the inertial boundary layer is located. Moreover, in this region the width of the bands is somewhat larger than found for the tails in the eastern part. This can be explained by an increase of the local Rhines scale, which scales with the rms velocity that is higher near the western boundary, resulting in flow structures with larger size. This makes the band structure somewhat irregular, as it has to deal with the increase in width (most visible for $\beta=400)$. Note that the cell at the southern boundary (near the bottom-left corner of the domain) is anticyclonic and the one near the northern boundary (near the topleft corner) is cyclonic, opposite the Fofonoff solution.

The striking difference between the observed mean flow and the Fofonoff solution can be attributed to the no-slip boundary conditions. LaCasce ${ }^{18}$ finds a mean flow consisting of a dual-gyre structure on a square domain with free-slip boundary conditions. The stucture is not identical to the Fofonoff solution, as the gyres are not filling the whole domain. The simulations by LaCasce ${ }^{18}$ have also shown that the constriction of the gyres to the northern and southern boundary becomes stronger if $\beta$ is increased. Wang and Vallis ${ }^{28}$ have shown how the Fofonoff solution emerges from a freely evolving random initial potential vorticity field in the inviscid case, and later on they have investigated the perturbation of the Fofonoff solution by the presence of a small amount of viscosity. The formation of a Fofonoff solution is found to be highly dependent on the type of boundary conditions. If a small viscosity is present and no-slip boundaries are applied, no mean flow was found during the decay of the flow. Our simulations differ from the simulations by Wang and Vallis, as forcing is applied and a statistically stationary turbulent flow field emerges. However, the mean flow we find is not very strong, which might explain the absence of observations of a mean flow in decaying simulations.

As solutions based on statistical mechanical predictions of the equilibrium state of solutions of the Euler equations (with the presence of the $\beta$ effect) reveal the existence of a linear relation between the mean streamfunction, $\bar{\psi}$ and the mean potential vorticity $\bar{q}$, it is apt to investigate the scatter plots of these quantities, $\bar{q}(\bar{\psi})$ [Figs. 9(a) and 9(b)]. Before investigating the scatter plots, note that the mean potential vorticity is mainly dominated by the background vorticity contribution $\beta_{0} y$, as is clearly illustrated in Fig. 9(c). Close to the boundary there is an additional significant contribution to the potential vorticity by the vorticity in the boundary layer. Figure 9(b) presents the scatter plot for points on the vertical line in the middle of the domain $(x=0)$. The alternations in the streamfunction can be directly correlated to the band structure of the mean flow, as the mean potential vorticity is linearly changing with $y$. Remember that the streamfunction on the boundary has to be zero, which is visible in the vertical ending tails. The variation in potential vorticity in these tails is due to the strong vorticity in the viscous boundary layers. The scatter plot for the whole domain shows the same structure, but is contaminated by the relatively strong contributions of the vortical structures in the inertial boundary layer. For instance, the big negative lobe is related to the strong cell at the position $(x, y)=(-0.8,0.5)$ in Fig. 8(a).

The relation between the potential vorticity and the streamfunction is, in our observations, clearly not linear. LaCasce has only found a linear relation inside the two gyres, while in the core of the gyres the streamfunction is nearly zero. ${ }^{18}$ Wang and Vallis have studied the effect of a number of different boundary conditions: free-slip, no-slip, and super-slip (where the normal vorticity gradient must be zero at the wall). The super-slip boundary condition is designed to minimize the effects of frictional boundary layers, while circulation is still conserved as for no-slip boundary conditions. $^{29}$ They found that only the numerical simulations with super-slip boundaries give a flow that closely resembles the Fofonoff solution. ${ }^{28}$ This is, however, for geostrophic flows with a relatively small $\beta$ number $(\beta=63)$. If $\beta$ is increased, the gyres are meridionally restricted to a region close to the northern and southern boundary. In the gyres the potential vorticity is homogeneous, while in the core region the streamfunction is zero. The sine-like relation between the potential vorticity and streamfunction we obtained for the no-slip boundary conditions does not resemble the relation found for either free-slip or super-slip boundary conditions. 


\section{SCALING OF THE BOUNDARY-LAYER THICKNESS}

In order to reduce the velocity to zero at the no-slip wall, a viscous boundary layer with a certain thickness $\delta_{v}$ is formed, which is characterized by high-velocity gradients in order to match the large velocities in the interior domain with the no-slip constraint at the boundary. In this section we will discuss the scaling of the viscous boundary-layer thickness that is needed to match the basin modes (derived for a freeslip bounded square domain) with the no-slip boundary condition in the present investigation. Subsequently, the obtained scaling relation is used to derive the scaling of the average boundary-layer thickness in geostrophic turbulence on a bounded domain with no-slip walls.

The viscous boundary layer associated with the basin modes will be modeled similar to the flow adjacent to a flat, oscillating no-slip plate, which is known as Stokes' second problem. The $x$ component of the flow above an infinite, horizontally oscillating plate can be found to obey (e.g., Ref. 6) $u(y, t)=U_{0} \exp \left(-y / \delta_{v}\right) \cos \left(\sigma t-y / \delta_{v}\right)$, where $U_{0}$ denotes the velocity amplitude, $\sigma$ the frequency of the oscillation, and $y$ the coordinate axis perpendicular to the plate. The viscous penetration depth $\delta_{v}$ is introduced as

$$
\delta_{\nu}=\sqrt{\frac{2 \nu}{\sigma}} .
$$

In the simulated flow fields, the high velocity gradients within the boundary layers are represented by a local, relatively high enstrophy $\left(\equiv \frac{1}{2} \omega^{2}\right)$. In order to quantify this concentration of enstrophy near the no-slip boundaries, the meridionally and the zonally line-integrated enstrophy are calculated by $I_{y}(x)=\frac{1}{2} \int_{-1}^{1} \omega^{2}(x, y) d y$ and $I_{x}(y)=\frac{1}{2} \int_{-1}^{1} \omega^{2}(x, y) d x$, respectively. As an example, the western boundary-layer thickness $\delta$ is determined by the perpendicular distance from the western boundary, where the lineintegrated enstrophy $I_{y}(x)$ is half of its maximum value at the western boundary. The thickness of the northern, eastern, and southern boundary layers, respectively, has been determined in a similar way. Since the flow is subject to westwardpropagating Rossby waves, an instantaneous flow field might lead to unreliable results, especially for the eastern and western boundaries. For this reason we have used a time average of squared vorticity to calculate $I_{y}(x)$ and $I_{x}(y)$. Using $I_{y}(x)$ and $I_{x}(y)$, the boundary-layer thickness of several released basin modes have been evaluated and compared with the model of the oscillating plate. Therefore, the basin mode frequency (7) is substituted in (16), leading to a dimensionless boundary-layer thickness of

$$
\delta_{n m}=\sqrt{\frac{4 \pi \nu\left(n^{2}+m^{2}\right)^{1 / 2}}{\beta_{0} L_{\mathcal{D}}}} .
$$

The simulations have been performed with $\beta$ ranging from 100 to 800 and $\nu^{\prime}$ ranging from $10^{-3}$ to $10^{-4}$. The consistency of the numerical results with the scaling indicated by Eq. (17), $\delta_{n m} \propto \sqrt{\nu / \beta_{0}}$, presented in Fig. 10, reveals that the model of the oscillating plate serves as an appropriate model for boundary layers induced by basin modes in a square domain with no-slip walls. Note that the boundary-layer

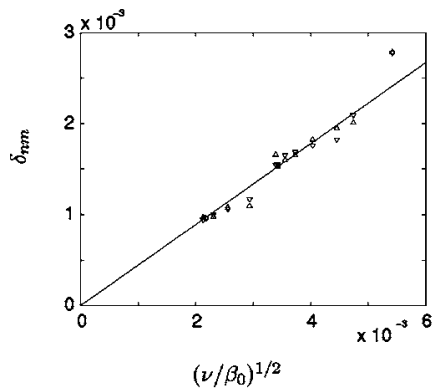

(a) $(n, m)=(1, m)_{m=1,2,3}$

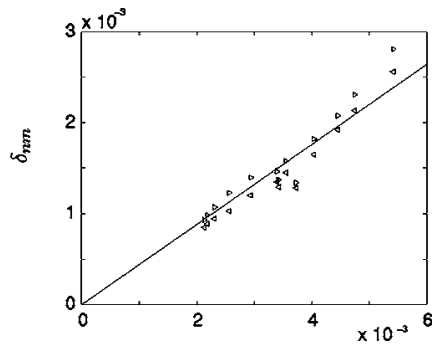

$\left(\nu / \beta_{0}\right)^{1 / 2}$

(b) $(n, m)=(2, m)_{m=1,2,3}$
FIG. 10. The boundary-layer thickness as a function of $\nu^{1 / 2} \beta_{0}^{-1 / 2}$ for six different basin modes on a no-slip domain. The data are arranged as follows: (a) $(n, m)=(1, m)_{m=1,2,3}$ and (b) $(n, m)=(2, m)_{m=1,2,3}$. In both panels, the measured boundary-layer thickness $\delta_{\nu}$ is indicated with the symbol + for $m=1, \emptyset$ for $m=2$, and $*$ for $m=3$. The theoretical boundary-layer thickness (17) (including a factor $\ln (2) / 2$ ) has also been plotted for $m=1$ (solid), $m=2$ (dashed), and $m=3$ (dotted).

thickness obtained from the numerical simulations deviates from the theory only for small values of $\beta$ and large viscosities. Deviations are also more pronounced for higher-order basin modes, as these modes have a smaller frequency for a given $\beta$.

Now Eq. (16) has been validated for the individual basin modes; it is used to estimate the boundary-layer thickness in the turbulence simulations. Therefore, the same procedure to predict the scaling behavior of the boundary-layer thickness has been used for the simulations of forced geostrophic turbulence, in which simultaneously many basin modes are present. The Rhines-scale-related shift in the excited basin modes, as was seen in the frequency spectra of Fig. 6, suggests that the Rhines scale could be involved in finding the mean frequency of the flow. Inserting the cutoff frequency (15) in (16) leads to a boundary-layer thickness that scales as $\delta_{\nu} \propto \nu^{1 / 2} \beta_{0}^{-1 / 4}$. In Fig. 11, this scaling theory is compared with results from the numerical simulations. As can be observed, the numerical data are in reasonable agreement with the theoretical prediction.

\section{CONCLUSION}

On a domain enclosed by no-slip boundaries, forced 2-D turbulence subject to the $\beta$ effect have been simulated. With-

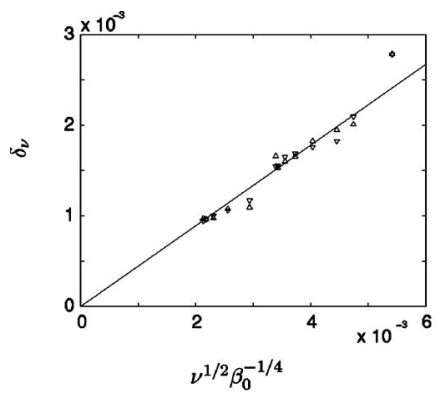

(a) northern and southern boundary

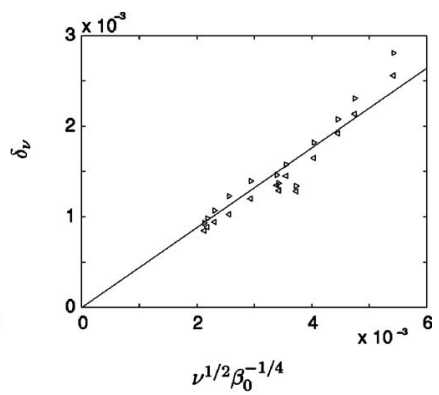

(b) eastern and western boundary
FIG. 11. The boundary-layer thickness as function of $\nu^{1 / 2} \beta_{0}^{-1 / 4}$ for (a) the northern and southern boundary and (b) the eastern and western boundary. The triangles are pointing to the boundary at which the data are taken. The solid, straight lines are the proportionality fits through the data points. 
out the presence of a $\beta$ plane a large, coherent vortex structure arises, as a direct result of the inverse energy cascade. If a $\beta$ plane is present, it is observed that the energy cascade is clearly impeded by the presence of the $\beta$ effect. This is explained by the Rhines scale: when the growing flow scales reach sizes comparable with the Rhines scale, the $\beta$ effect becomes dynamically more important than advection and, consequently, the Rossby wave mechanism takes over from the inverse energy cascade mechanism.

Frequency spectra of the flow profoundly exhibit the existence of basin modes in forced 2-D turbulence in a square domain with no-slip boundaries. For the $\beta$ numbers used, the influence of the no-slip walls on the basin modes appears to be restricted to very thin boundary layers, while the basin mode frequency and shape are well described by solutions of inviscid flows in square bounded domains with free-slip boundaries. As $\beta$ increases, the higher-order basin modes are more dominantly present, which is in agreement with a decreasing Rhines scale. For structures with scales larger than the Rhines scale, Rossby wave propagation is favored above the organizing mechanism associated with the inverse cascade. Hence, basin modes with length scales around the Rhines scale are favored. The presence of basin modes also causes the formation of an inertial boundary near the western boundary.

The mean flow found in the turbulence simulations shows a zonal band structure that resembles the flow on a periodic domain (although the kinetic energy of the mean flow represents only $2 \%-4 \%$ of the kinetic energy of the turbulent flow field itself). This is a rather surprising result, as numerous other simulations show a mean flow consisting of a dual gyre on a closed domain. The difference can be attributed solely to the applied no-slip boundary conditions in the present investigation. There is a sine-like relation between the mean potential vorticity $\bar{q}$ and the mean stream function $\bar{\psi}$, which is directly related to the band structure observed for the mean flow.

For the basin-mode-dominated flow, a boundary-layer model was derived from the problem of an oscillating plate (Stokes' second problem). Relaxations of individual basin modes have confirmed that this theory provides an appropriate model for describing the boundary layers, with the boundary-layer thickness scaling as $\delta_{\nu} \propto \sqrt{\nu / \beta_{0}}$. The continuing propagation of basin modes keeps the boundary layers very thin, and prevents detachment from the boundary by advection. Based on the frequency correlated with the Rhines wave number, a boundary-layer scaling was derived and validated for forced turbulent flows on the $\beta$ plane: $\delta_{\nu} \propto \nu^{1 / 2} \beta_{0}^{-1 / 4}$.

\section{ACKNOWLEDGMENTS}

One of the authors (W.K.) was supported by the Computational Science Program (Project No. 635.000.002) with financial aid from the Netherlands Organization for Scientific Research (NWO).
${ }^{1}$ R. H. Kraichnan, "Inertial ranges in two-dimensional turbulence," Phys. Fluids 10, 1417 (1967).

${ }^{2}$ M. Hossain, W. H. Matthaeus, and D. Montgomery, "Long-time state of inverse cascades in the presence of a maximum length scale," J. Plasma Phys. 30, 479 (1983).

${ }^{3}$ L. M. Smith and V. Yakhot, "Bose condensation and small-scale structure generation in a random force driven 2D turbulence," Phys. Rev. Lett. 71, 352 (1993).

${ }^{4} \mathrm{G}$. K. Batchelor, "Computation of the energy spectrum in homogeneous two-dimensional turbulence," Phys. Fluids Suppl. II 12, 223 (1969).

${ }^{5}$ J. Pedlosky, Geophysical Fluid Dynamics (Spinger-Verlag, Berlin, 1987).

${ }^{6}$ P. K. Kundu, Fluid Mechanics (Academic, New York, 1990).

${ }^{7}$ A. E. Gill, Atmosphere-Ocean Dynamics (Academic, New York, 1982).

${ }^{8}$ C. G. Rossby, "On the propagation of frequencies and energy in certain types of oceanic and atmospheric waves," J. Meteorol. Soc. Jpn. 2, 187 (1945).

${ }^{9}$ P. B. Rhines, "Waves and turbulence on the beta-plane," J. Fluid Mech. 69, 417 (1975).

${ }^{10}$ G. P. Williams, "Planetary circulations: 2. The Jovian quasi-geostrophic regime," J. Atmos. Sci. 36, 932 (1994).

${ }^{11}$ T. Nozawa and S. Yoden, "Formation of zonal band structures in forced two-dimensional turbulence on a rotating sphere," Phys. Fluids 9, 2081 (1997).

${ }^{12}$ W. H. Matthaeus and D. Montgomery, "Selective decay hypothesis at high mechanical and magnetic Reynolds numbers," Ann. N.Y. Acad. Sci. 357, 203 (1980).

${ }^{13}$ J. C. McWilliams, "The emergence of coherent vortices in turbulent flow," J. Fluid Mech. 146, 21 (1984).

${ }^{14}$ P. B. Rhines, "Geostrophic turbulence," Annu. Rev. Fluid Mech. 11, 401 (1979).

${ }^{15} \mathrm{H}$. Huang and W. A. Robinson, "Two-dimensional turbulence and persistent zonal jets in a global barotropic model," J. Atmos. Sci. 55, 611 (1998).

${ }^{16}$ M. S. Longuet-Higgins, "Planetary waves on a rotating sphere," Proc. R. Soc. London, Ser. A 279, 446 (1964).

${ }^{17}$ H. J. H. Clercx and G. J. F. van Heijst, "Energy spectra for decaying 2D turbulence in a bounded domain," Phys. Rev. Lett. 85, 306 (2000).

${ }^{18}$ J. H. LaCasce, "On turbulence and normal modes in a basin," J. Mar. Res. 60, 431 (2002).

${ }^{19}$ H. J. H. Clercx, "A spectral solver for the Navier-Stokes equations in the velocity-vorticity formulation for flows with two nonperiodic directions," J. Comput. Phys. 137, 186 (1997).

${ }^{20}$ C. Canuto, M. Y. Hussaini, A. Quarteroni, and T. A. Zang, Spectral Methods in Fluid Dynamics (Spinger-Verlag, Berlin, 1987).

${ }^{21}$ D. K. Lilly, "Numerical simulations of two-dimensional turbulence," Phys. Fluids 12, 240 (1969).

${ }^{22}$ M. E. Maltrud and G. K. Vallis, "Energy spectra and coherent structures in forced two-dimensional turbulence and beta-plane turbulence," J. Fluid Mech. 228, 321 (1991).

${ }^{23}$ H. J. H. Clercx, S. R. Maassen, and G. J. F. van Heijst, "Spontaneous spin-up during the decay of 2D turbulence in a square container with rigid boundaries," Phys. Rev. Lett. 80, 5129 (1998).

${ }^{24}$ H. J. H. Clercx, A. H. Nielsen, D. J. Torres, and E. A. Coutsias, "Twodimensional turbulence in square and circular domains with no-slip walls," Eur. J. Mech. B/Fluids 20, 557 (2001).

${ }^{25}$ D. Molenaar, H. J. H. Clercx, and G. J. F. van Heijst, "Spin-up of angular momentum in forced 2D turbulence in a square Dirichlet domain," Physica D 196, 329 (2004).

${ }^{26}$ N. P. Fofonoff, "Steady flow in a frictionless homogeneous ocean," J. Mar. Res. 13, 254 (1954).

${ }^{27}$ R. Salmon, H. G. Holloway, and M. C. Hendershott, "The equilibrium statistical mechanics of simple quasigeostrophic models," J. Fluid Mech. 75, 691 (1976).

${ }^{28}$ J. Wang and G. K. Vallis, "Emergence of Fofonoff states in inviscid and viscous ocean circulation models," J. Mar. Res. 52, 83 (1994).

${ }^{29}$ J. C. Marshall, "Eddy-mean flow interaction in a barotropic ocean model," Q. J. R. Meteorol. Soc. 110, 573 (1984). 